Provided for non-commercial research and education use. Not for reproduction, distribution or commercial use.

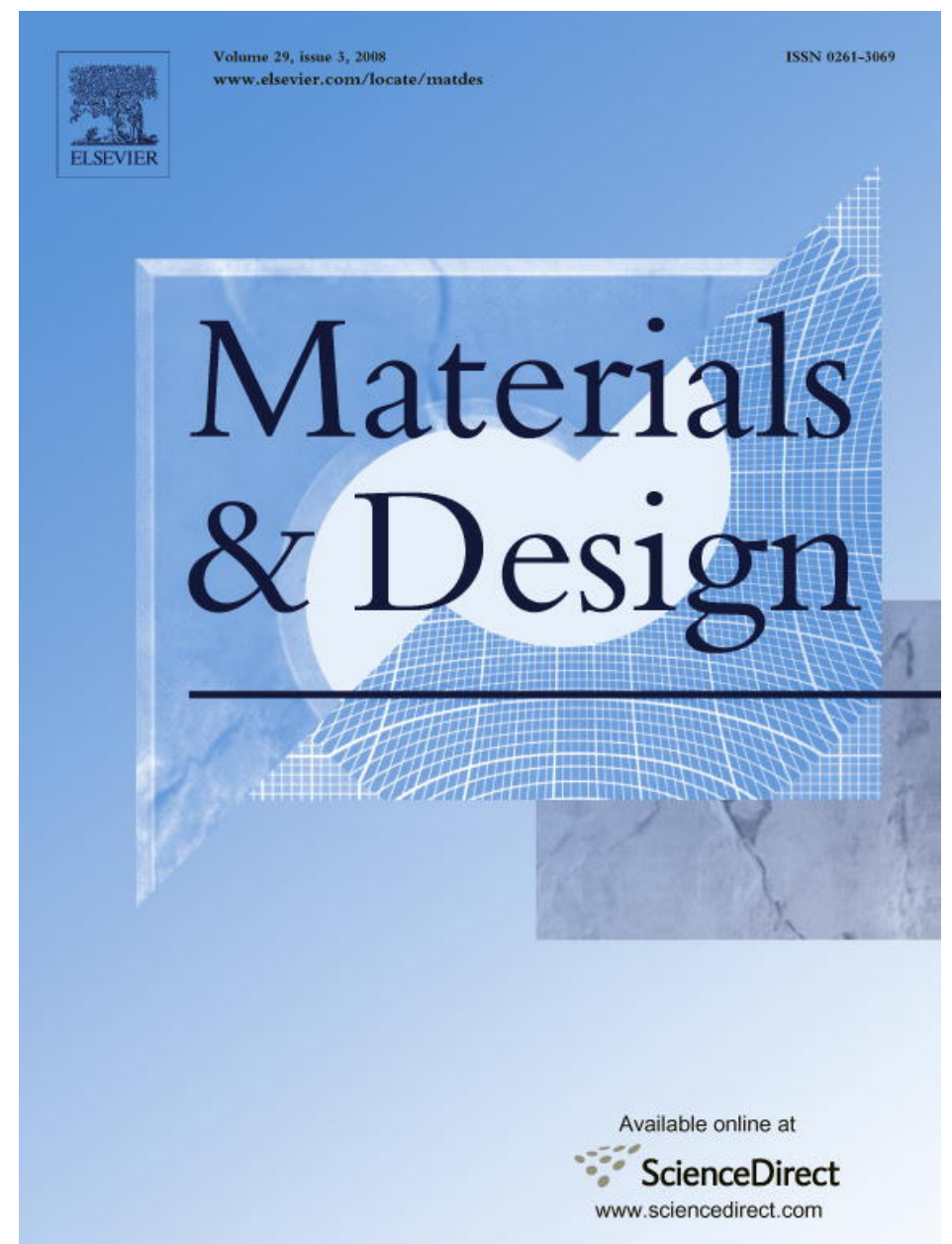

This article was published in an Elsevier journal. The attached copy

is furnished to the author for non-commercial research and education use, including for instruction at the author's institution, sharing with colleagues and providing to institution administration.

Other uses, including reproduction and distribution, or selling or licensing copies, or posting to personal, institutional or third party websites are prohibited.

In most cases authors are permitted to post their version of the article (e.g. in Word or Tex form) to their personal website or institutional repository. Authors requiring further information regarding Elsevier's archiving and manuscript policies are encouraged to visit: 


\title{
Modeling the progressive axial crushing of foam-filled aluminum tubes using smooth particle hydrodynamics and coupled finite element model/smooth particle hydrodynamics
}

\author{
L. Aktay ${ }^{a}$, A.F. Johnson ${ }^{\text {a }}$, A.K. Toksoy ${ }^{\text {b }}$, B.-H. Kröplin ${ }^{\text {c }}$, M. Güden ${ }^{\text {b,d,* }}$ \\ a German Aerospace Center, Institute of Structures and Design, Pfaffenwaldring 38-40, 70569 Stuttgart, Germany \\ ${ }^{\mathrm{b}}$ Department of Mechanical Engineering, İzmir Institute of Technology, Gulbahce, Urla, Izmir 35430, Turkey \\ ${ }^{c}$ Institute of Statics and Dynamics of Aerospace Structures, University of Stuttgart, Pfaffenwaldring 27, 70569 Stuttgart, Germany \\ ${ }^{\mathrm{d}}$ Center for Materials Research, Izmir Institute of Technology, Gulbahce, Urla, Izmir 35430, Turkey \\ Received 6 November 2006; accepted 7 March 2007 \\ Available online 27 March 2007
}

\begin{abstract}
As alternatives to the classical finite element model (FEM), a meshless smooth particle hydrodynamics (SPH) method, in which the discrete particles represent a solid domain, and a coupled FEM/SPH modeling technique were investigated for the numerical simulation of the quasi-static axial crushing of polystyrene foam-filled aluminum thin-walled aluminum tubes. The results of numerical simulations, load-deformation histories, fold lengths and specific absorbed energies, were found to show satisfactory correlations with those of experiments and FEM. The results further proved the capabilities of the SPH Method and coupled FEM/SPH modeling technique in predicting the crushing behavior of foam-filled thin-walled tubes.
\end{abstract}

(C) 2007 Elsevier Ltd. All rights reserved.

Keywords: Energy absorption; Smooth particle hydrodynamics; Coupling phenomena; Foam-filled tubes; Polystyrene foam

\section{Introduction}

Crashworthiness and impact resistance are challenging engineering problems directly related to structural integrity and passenger safety. European Community Road Accident Database [1] has shown that the number of accidents has the same tendency between 1991 and 2003 and the number of injuries remained almost the same. However, the number of casualties decreased approximately $30 \%$, showing an effective and increased use of passive protection safety systems (seat belts, airbags and etc.) and the active protection safety systems (sensors, anti-lock brakes and etc.). The vital importance of the crashworthiness has moti-

\footnotetext{
${ }^{*}$ Corresponding author. Address: Department of Mechanical Engineering, İzmir Institute of Technology, Gulbahce, Urla, Izmir 35430, Turkey. Tel.: +90 232 7507816; fax: +90 2327507890 .

E-mail address: mustafaguden@iyte.edu.tr (M. Güden).
}

vated researchers to concentrate on the designing of novel structural components that are capable of absorbing the crash energy in a controlled manner. Columnar structures including square and circular metal tubes are the examples to such components progressively diminishing the large amount of crash energy through plastic collapse. Since the pioneer work of Alexander [2], many experimental and numerical investigations have been carried out on the crushing behavior of columnar structures and some of them have been reviewed by Alghamdi [3]. The recent focus has been on the use of various material combinations in order to enhance the specific absorbed energy (SAE) of these structures.

Thornton [4] showed that although polyurethane foam filling increased the SAE values of the thin-walled sections, it was not weight-effective when compared with the thickening of the tube wall. Reid et al. [5] investigated the crushing mechanism of polyurethane foam-filled metal 
tubes at quasi-static and dynamic deformation rates. The interaction between the tube wall and the foam filler was found to result in a tendency for the concertina mode of deformation. Seitzberger et al. [6] focused on foam-filled monolithic and bitubular arrangements including square, hexagonal and orthogonal tubes and noted that these structural arrangements should also be taken into account in the designing of efficient absorbing components with foam-filled metal tubes. Santosa and Wierzbicki [7] investigated the crushing behavior of aluminum honeycomb and foam-filled box columns numerically and experimentally and showed that the effect of filler on the tube crushing load was similar when the strong axis of the honeycomb was through and normal to the compression axis, proving that both axial and lateral strengths of the filler were effective in increasing the crushing load of the tube. Further, Santosa et al. [8] noted that the bonding between filler and tube wall increased the average crushing load of filled tube over the unbounded filled tube when appropriate tube geometry and foam density were chosen. Aktay et al. [9] investigated the quasi-static crushing of extruded polystyrene foam-filled aluminum thinwalled tubes. The numerical model satisfactorily predicted the deformation mode switch in the foam-filled tubes that primarily depended on the tube diameter and the foam density. Polystyrene foams were also investigated as a crush energy absorber element in different case studies including motorcyclist's helmets inner material for preventing brain damage and race track barrier material for the distribution of the energy generated during the crash of race cars $[10,11]$.

Although experimental tests under crush conditions provide valuable information about the deformation characteristics of the tested material, the crush test programs are destructive, time consuming and consequently expensive for the industry. In order to reduce the development and certification costs, computational methods are generally used to predict structural integrity and energy absorption under impact loading conditions. The finite element model (FEM) is an established effective tool for predicting structural behavior at different loading conditions; however, it is less reliable in large deformations and structural failures under dynamic loads. Furthermore, since the FEM is based on the continuum mechanics formulations requiring element connectivity, it is difficult to simulate the failures involving material fragmentation. Alternatively, meshless methods have been developed and applied to numerical simulations involving material failure and damage. Meshless methods replace finite elements by a set of nodes or particles within the problem domain and its boundaries. The mesh connectivity is therefore not critical as in the FEM. Smooth particle hydrodynamics (SPH) is one of the earliest particle methods in computational mechanics, developed by Lucy [12] and Gingold and Monaghan [13] in order to solve the astrophysical problems in 3D open space. Since its invention SPH has been extended to the simulations of dynamic loadings and metal forming processes [14-17]. The SPH however uses a computationally expensive dynamic neighboring search algorithm and consequently coupling methods have been proposed to improve the efficiency of meshless methods [18]. Johnson [19] developed a coupling technique based on the master-slave contact algorithm for the interaction between finite elements and particles and simulated the penetrations of rigid projectiles into aluminum and steel plates where FEM suffered from severe element distortion. Attaway et al. [20] studied the coupling of SPH with transient dynamics FE code PRONTO using a contact-like coupling algorithm. Sauer et al. [21] noted the numerical instabilities in the central processing unit (CPU) intensive SPH method and applied moving least squares correction methodology to improve the consistency of the meshless SPH approximation. Hiermeier et al. [22] employed an adaptive node splitting with adaptive coupling methodology to model the crushing behavior of a carbon fiber reinforced plastic. It was concluded that the computational time was greatly reduced as compared with pure SPH discretization [22]. De Vuyst et al. [23] have recently presented a contact based algorithm allowing a frictionless sliding contact between finite elements and particles and simulated a projectile impact to an aluminum target and a ditching problem between a cylinder and water surface. Such coupling methods would exploit the potential of each method while avoiding their deficiencies.

In this study, a meshless SPH method and an FEM/SPH coupling technique were used to model polystyrene foam filler deformation in a foam-filled aluminum circular tube as alternatives to FEM. In the coupled FEM/SPH technique, discrete particles were used to model only the deformation zones of severe element distortion which cause hour-glassing and tube wall penetration, while the major part of the polystyrene foam filler was modeled with finite elements. The numerical results including deformation modes, load-deformation histories, fold length and SAE values were compared with the results of experimental and FEM numerical analysis.

\section{Experiments and modeling}

\subsection{Materials and testing}

Commercially available extruded polystyrene foam sheets, manufactured by Izocam Company of Turkey, with a density of $27.8 \mathrm{~kg} \mathrm{~m}^{-3}$ were used as filler. The foam filler samples were core-drilled and then tightly inserted (without adhesive) into thin-walled empty Al (99.7\%) tubes, $25 \mathrm{~mm}$ in outer diameter, $0.29 \mathrm{~mm}$ in wall thickness and $40 \mathrm{~mm}$ in length, without imposing any damage to the cells (Fig. 1). The yield and ultimate tensile strength of the tube material were determined in accordance with ASTM B557M as $112 \mathrm{MPa}$ and $170 \mathrm{MPa}$, respectively. The compression behavior of the filler material was determined in accordance with ASTM 1621-91. Compression tests were performed using computer controlled Schmadzu AGI universal test machine without any constraint applied to the ends of tubes at a deformation rate of $100 \mathrm{~mm} \mathrm{~min}^{-1}$. The compression stress-strain curve of the filler shows a typical elastic-plastic material response as shown in Fig. 2. 

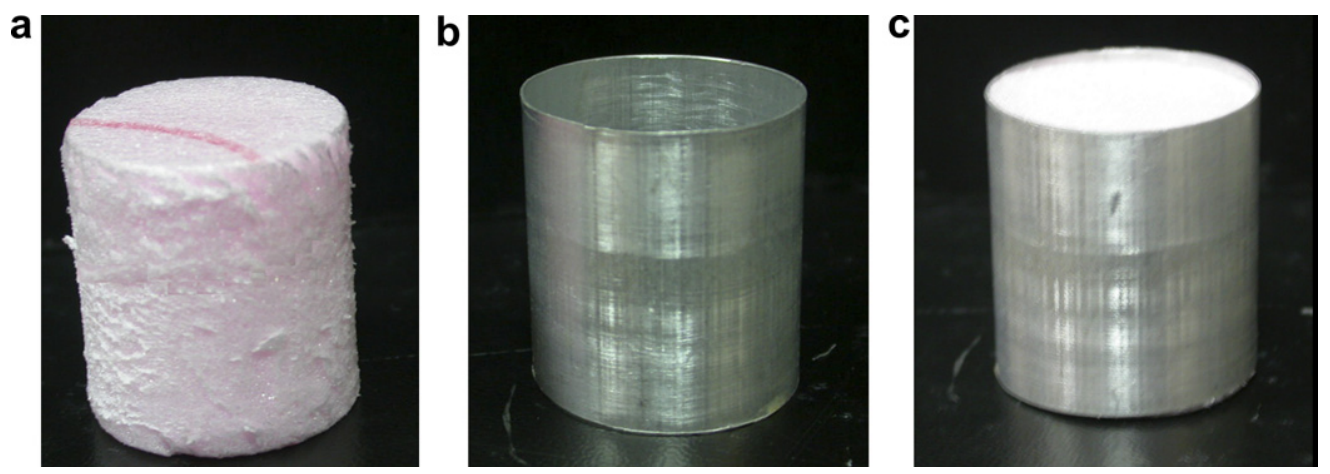

Fig. 1. Images of (a) foam filler, (b) empty aluminum tube and (c) foam-filled aluminum tube.

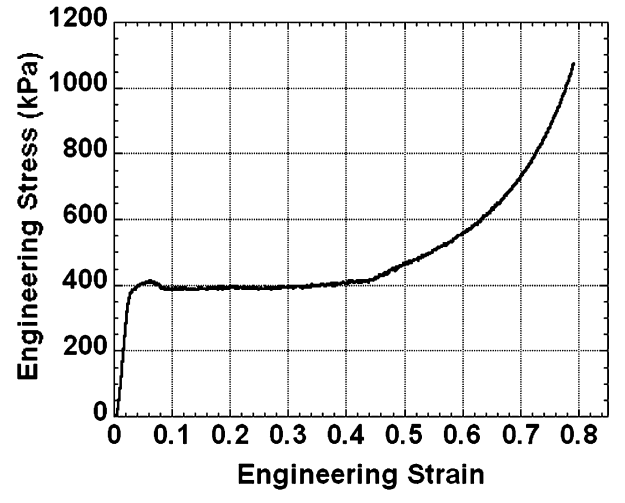

Fig. 2. Quasi-static compression stress-strain curve of foam filler.

\subsection{Polystyrene foam modeling with solid element}

Finite element simulations were carried out using the explicit finite element code PAM-CRASH ${ }^{\text {TM }}$ and visualization was done by PAM-VIEW ${ }^{\text {TM }}$ in post-processing session. A crushable foam solid material model (Material Type 2) was used to simulate the compression deformation of the filler. Fig. 3 shows the FE discretization of the foam filler. The elastic response was described by the tangent and shear modulus. The inelastic material response was modeled using the pressure dependent von Mises $\left(J_{2}\right.$ plasticity) yield surface given as

$\phi_{S}=J_{2}-\left(a_{0}+a_{1} p+a_{2} p^{2}\right)=0$

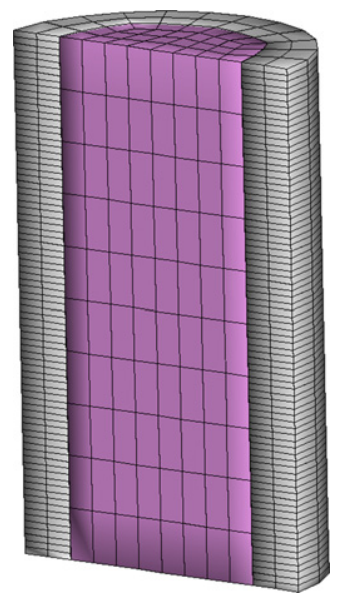

Fig. 3. FE discretization of foam filler. and

$J_{2}=\frac{1}{2} S_{i j} S_{i j}=\frac{1}{3} \sigma_{\mathrm{Y}}^{2}$

where $J_{2}$ is the second invariant of the deviatoric stress tensor of the von Mises yield surface, $a_{0}, a_{1}$ and $a_{2}$ are the material parameters and $p$ and $\sigma_{\mathrm{Y}}$ are the pressure and effective yield stress, respectively. The aluminum tubes and compression test plates were modeled using Belytschko-Tsay4 node-thin shell and 8-node solid elements, respectively. Detailed information about the FE modeling procedure is given in [9].

\subsection{Polystyrene foam modeling with discrete particles}

SPH method is based on two interpolation approximations: kernel and particle approximation. Considering the function $f(x)$ in Eq. (3), the value at a point of $f(x)$ over the domain $\Omega$ could be extracted from its integral using the delta $(\delta)$ function given in Eq. (4) as a filter

$\langle f(\bar{x})\rangle=\int_{\Omega} f\left(\bar{x}^{\prime}\right) \delta\left(\bar{x}-\bar{x}^{\prime}\right) \mathrm{d} \bar{x}^{\prime}$

and

$\int_{\Omega} \delta\left(\bar{x}-\bar{x}^{\prime}\right) \mathrm{d} \bar{x}^{\prime}=1$

As $h \rightarrow 0, \delta\left(\bar{x}-\bar{x}^{\prime}\right)$ can be replaced with a kernel function of $W\left(\bar{x}-\bar{x}^{\prime}, h\right)$, which has a support domain determined by the parameter $h$,

$\lim _{h \rightarrow 0} W\left(\bar{x}-\bar{x}^{\prime}, h\right)=\delta\left(\bar{x}-\bar{x}^{\prime}\right)$

Inserting Eq. (5) into Eq. (3) gives the following equation:

$\langle f(\bar{x})\rangle=\int_{\Omega} f\left(\bar{x}^{\prime}\right) W\left(\bar{x}-\bar{x}^{\prime}, h\right) \mathrm{d} \bar{x}^{\prime}$

Since domain is represented by discrete particles, the summation of the contributions of each particle within the kernel approximation range gives the smoothed value of $f(x)$ at a point (particle approximation) as

$\langle f(\bar{x})\rangle=\sum_{j=1}^{N}\left(\frac{m_{j}}{\rho_{j}}\right) f_{j} W\left(\left|\bar{x}-\bar{x}^{\prime}\right|, h\right)$

where $N$ represents the number of discrete particles and $m_{j}$ and $\rho_{j}$ are the mass and density of the particle $j$, respectively. Localization and information transformation from one particle to another are achieved through an interpolation distance called the smoothing length. The sphere of influence of a particle is a multiple of its smoothing length.

The fully discrete particle and combined solid element/discrete element model of the filler in the foam-filled tube are shown sequentially in Figs. 4 and 5. Discrete particles and solid elements coupled with kinematical type tied contact algorithm that allows the stable computations without distortion. The material model used for discrete SPH particles was an isotropicelastic-plastic-hydrodynamics solid material model, of which an equation of state was used to model the pressure-volume relation as 
a

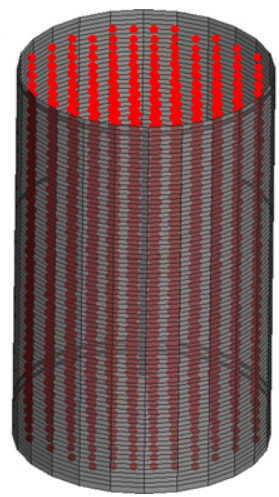

b

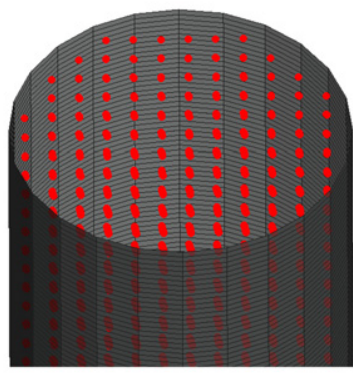

Fig. 4. Discrete particle modeling of foam filler: (a) general and (b) detail view.
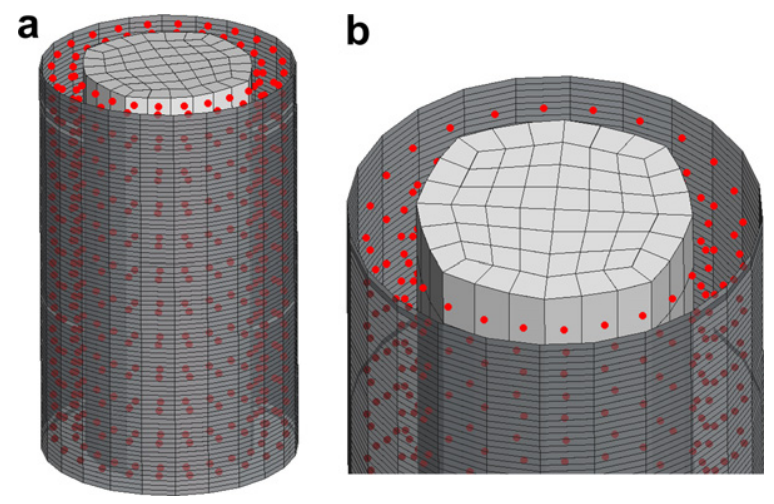

Fig. 5. Coupled solid element/discrete particle modeling of foam filler: (a) general and (b) detail view.

$p=C_{0}+C_{1} \mu+C_{2} \mu^{2}+C_{3} \mu^{3}$

where $C_{0}, C_{1}, C_{2}$ and $C_{3}$ are the material constants and $\mu$ is a dimensionless compressibility parameter which depends on the current density $(\rho)$ and initial density $\left(\rho_{0}\right)$ as,

$\mu=\frac{\rho}{\rho_{0}}-1$

The polynomial form of the equation is a widely used approximation for many materials and it reduces, when $C_{0}=C_{2}=C_{3}=0$, to a dilatational elastic material law with bulk modulus of $C_{1}$ [24]. Discrete particles were generated with a simple transformation of finite element mesh into mass points.

The optimum number of particles in SPH model and the number of solid elements and particles for coupled FEM/SPH approach have been investigated. The distance between the SPH particles kept constant to obtain a homogeneous distribution of interaction. Since the change in the number of particles resulting from the change of smoothing length affects the model results, the model was constructed in a way that the results were stable concerning the interaction between the particles. The same interaction parameters are used in SPH and coupled FEM/SPH modeling for the characterization of foam material.

\section{Results and discussion}

Experimental and FEM numerical deformation patterns of empty and foam-filled tubes at 50\% deformation are shown in Fig. 6a-d. Corresponding experimental and FEM load-displacement curves of empty and foam-filled tubes are shown in Fig. 7a and b, respectively. Although a
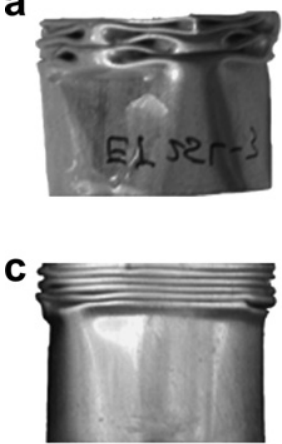

b
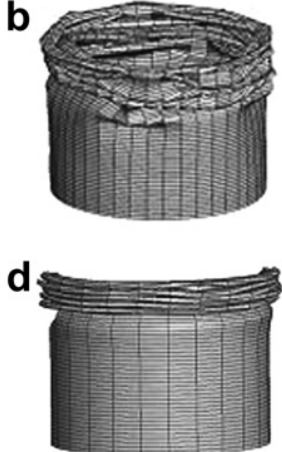

Fig. 6. Deformed structures at 50\% deformation (a) experimental empty tube, (b) numerical (FEM) empty tube, (c) experimental foam-filled tube and (d) numerical (FEM) foam-filled tube.

empty tube, both in experiments and numerical simulations, deformed in diamond mode (Fig. 6a and b), the foam filling was reverted the deformation mode from diamond to concertina as shown in Fig. 6c and d. The switch in the deformation mode was attributed to the wall thickening effect of foam filling [25]. It is noted that the deformed shapes and load-displacement curves of FEM show good agreement with the experimental results. As the foam filler experiences relatively large deformations, the sizes of solid elements become smaller, particularly in the region where the tube wall interacts with the filler. Since an explicit numerical scheme is used in the analysis, the small element
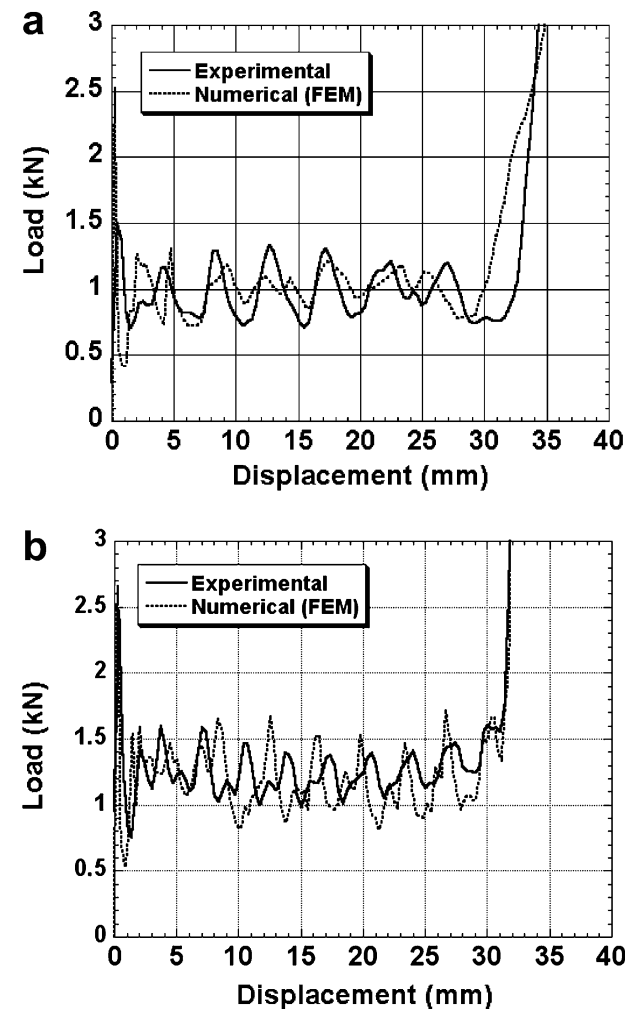

Fig. 7. Comparison of experimental and numerical load-displacement curves (a) empty and (b) foam-filled tube. 
a

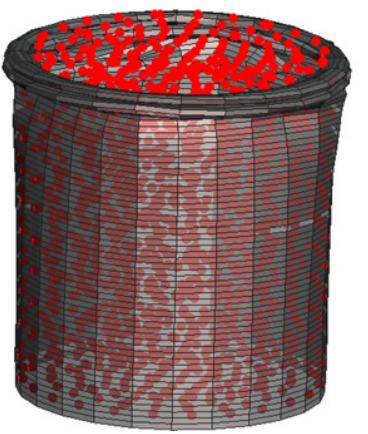

b

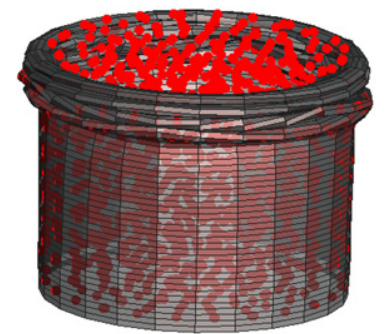

C

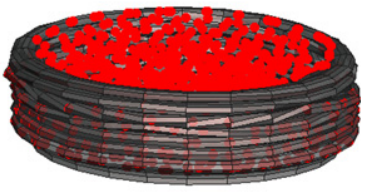

Fig. 8. SPH model progressive folding of foam-filled tube at (a) $30 \%$, (b) $50 \%$ and (c) $80 \%$ deformation.

a

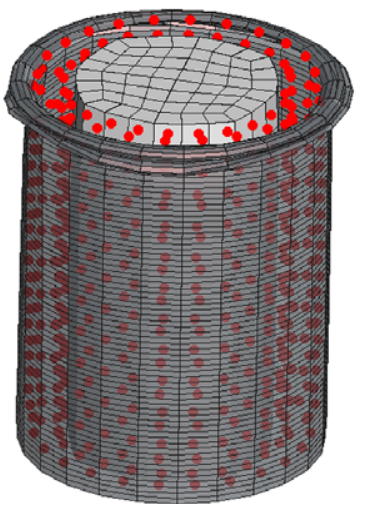

b

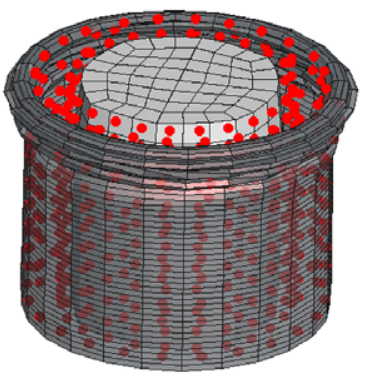

C

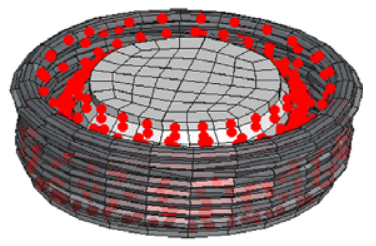

Fig. 9. Coupled FEM/SPH model progressive folding of foam-filled tube at (a) $30 \%$, (b) $50 \%$ and (c) $80 \%$ deformation.

size may cause expensive computational time and error termination.

Figs. 8 and 9 show SPH and coupled FEM/SPH simulated progressive foam-filled tube deformations at $30 \%$, $50 \%$ and $80 \%$ deformations, respectively. The models used show a deformation mode change from diamond in the empty tube into concertina in the filled tubes, similar to FEM. The change of deformation mode in SPH method and coupled FEM/SPH simulations further proved the successful employment of the interactions between shell elements and discrete particles and discrete particles and solid elements. The resultant load-displacements curves of SPH and coupled FEM/SPH simulations are shown in Fig. 10a and b, respectively. In the same figures, the experimental load-displacements curves are also shown for comparison. Both approaches, as also seen in these figures, are found to give satisfactory agreements with the experimental load-displacement curves. It is also noted in Fig. 10a and $\mathrm{b}$ and in Fig. $7 \mathrm{~b}$ that the proposed approaches result in relatively higher load values than those of FEM around the densification region of the tube deformation. The higher load values are however more pronounced in SPH method (Fig. 10a). Under extensive crushing, since the particles have interactions with the surrounding particles in addition to their initial neighbors, the densification of $\mathrm{SPH}$ modeled foam is steeper than that of FE modeled foam. The effect of smoothing length on the load-displace-
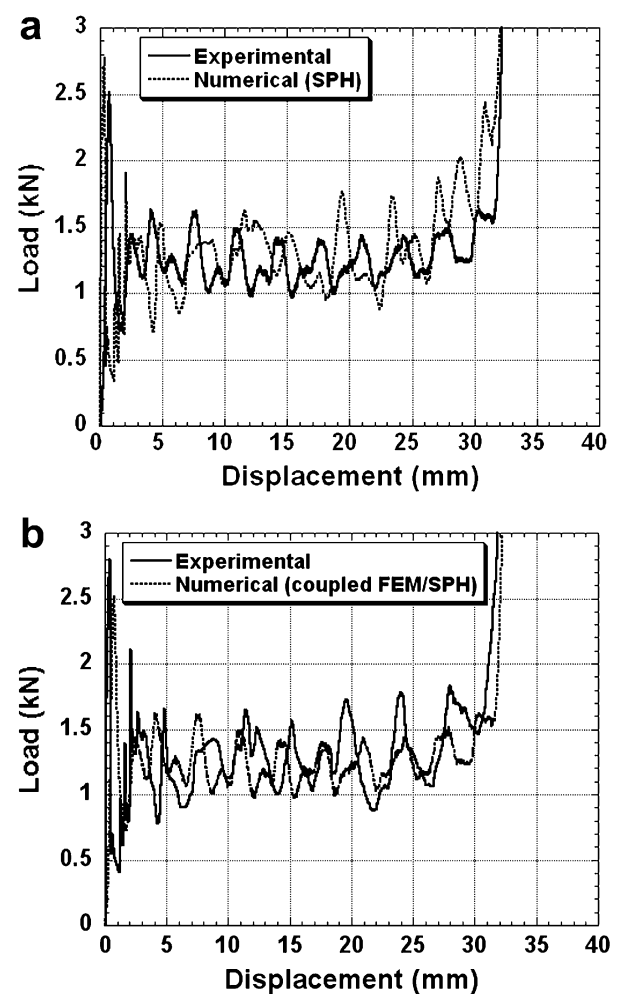

Fig. 10. Comparison of experimental and numerical load-displacement curves of foam-filled tube, (a) SPH and (b) coupled FEM/SPH. 

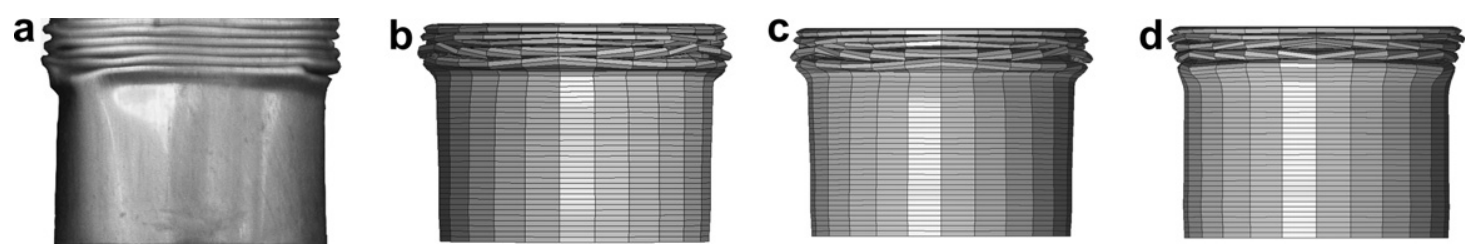

Fig. 11. Deformed shapes of foam-filled tubes at 50\% deformation: (a) experimental, (b) FEM, (c) SPH and (d) coupled FEM/SPH.

ment curves of foam-filled tubes will be further investigated in another study.

It was previously shown that the restraining effect of foam filling to the tube wall decreased the fold lengths hence increased the number of folds formed in the foamfilled tubes [9]. The experimental, FEM, SPH and coupled FEM/SPH deformed shapes of the foam-filled tubes are sequentially shown in Fig. 11a-d at 50\% deformation. The total number of folds formed, 5, in the models used, as seen in this figure, is the same with that of experiment. The numerically measured fold lengths are however found slightly higher than the experimentally measured fold lengths (Fig. 12). The foam filling causes experimentally $75 \%$ reduction in the fold length as compared with empty tube, while the reduction in the fold length increases to $77 \%, 78 \%$ and $84 \%$ in FEM, SPH and coupled FEM/ SPH simulations, respectively. The experimental and

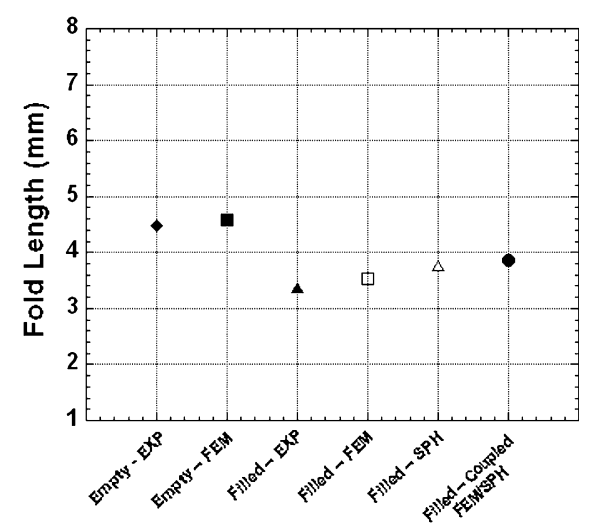

Fig. 12. Numerical and experimental fold lengths at $50 \%$ deformation.

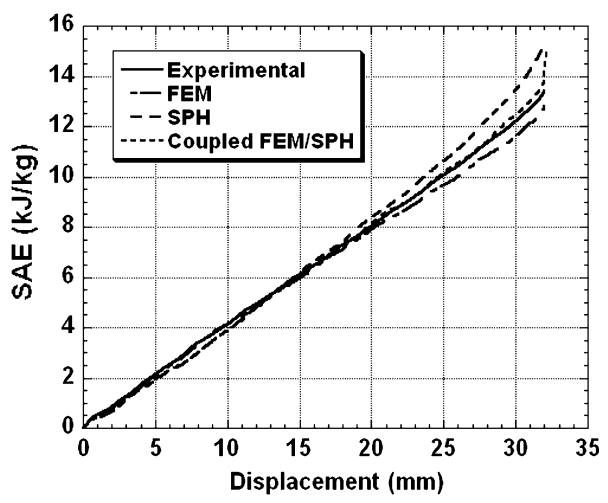

Fig. 13. Comparison of experimental and numerical SAE vs. displacement curves of foam-filled tube. numerical SAE values corresponding to $50 \%$ deformation of foam-filled tubes are shown in Fig. 13 for comparison. Numerical simulations based on SPH and coupled FEM/ SPH show comparable SAE values with those obtained by FEM and experiments. In filled tubes, the differences in SAE values at $50 \%$ deformation between experiment and FEM and SPH and coupled FEM/SPH are 2\%, 3.9\% and $1.1 \%$, respectively.

In FE modeling of foam filler using explicit numerical solution technique and classical solid elements, the time step depends on the size of the element in the simulation and the FE model has the longest simulation time primarily due to the severe solid elements deformation. The coupled model reduces the computational time by a factor of 0.75 compared to SPH model and by a factor of 0.30 compared to FE model. The internal force calculation consumes $78 \%$ of the total computational time in SPH model while it is reduced to $65 \%$ in coupled FEM/SPH technique.

\section{Conclusions}

The quasi-static axial crushing of polystyrene foamfilled aluminum tubes was simulated using SPH and coupled FEM/SPH and the numerical results were compared with those obtained using FEM and experiments. Both numerical methods were found to give satisfactory agreements with FEM and experiments. The used models successfully simulated the switch in the deformation mode of filled tubes. The comparable load-deformation histories, fold lengths and SAE values between the experiments and numerical analysis, further confirmed that both SPH and coupled FEM/SPH could be applied to model foam-filled tube deformation in cases where classical FEM suffers from severe element distortion and expensive computations.

\section{Acknowledgement}

The authors thank the Scientific and Technical Council of Turkey (TUBITAK) for the Grant \#MISAG-227.

\section{References}

[1] Annual Statistical Report 2005. CARE, European Commission Community Road Accident Database 2005.

[2] Alexander JM. An approximate analysis of collapse of thin-walled cylindrical shells under axial loading. Q J Mech Appl Math 1960;13:1-9.

[3] Alghamdi AAA. Collapsible impact energy absorbers: an overview. Thin-Walled Struct 2001;39:189-213. 
[4] Thornton PH. Energy absorption by foam-filled structures. SAE paper $1980,800372$.

[5] Reid SR, Reddy TY, Gray MD. Static and dynamic axial crushing of foam-filled sheet metal tubes. Int J Mech Sci 1986;23:295-322.

[6] Seitzberger M, Rammerstorfer FG, Gradinger R, Degischer HP, Blaimschein M, Walch C. Experimental studies on the quasi-static axial crushing of steel columns filled with aluminum foam. Int $\mathbf{J}$ Solids Struct 2000;37:4125-47.

[7] Santosa S, Wierzbicki T. Crash behavior of box columns filled with aluminum honeycomb or foam. Comput Struct 1998;68:343-67.

[8] Santosa S, Wierzbicki T, Hanssen AG, Langseth M. Experimental and numerical studies of foam-filled sections. Int $\mathbf{J}$ Impact Eng 2000;24:509-34.

[9] Aktay L, Toksoy AK, Güden M. Quasi-static crushing of extruded polystyrene foam-filled thin-walled aluminum tubes: Experimental and numerical analysis. Mater Design 2005;27:556-65.

[10] Lui D-S, Chang C-Y, Fan C-M, Hsu S-L. Influence of environmental factors on energy absorption degradation of polystyrene foam in protective helmets. Eng Fail Anal 2003;10:581-91.

[11] Bielenberg RW, Reid JD. Modeling crushable foam for the safer racetrack barrier. In: 8th International LS-DYNA User Conference, vol. 6. 2004. p. 1-8.

[12] Lucy LB. A numerical approach to the testing of the fission hypothesis. AJ 1977;82:1013-24.

[13] Gingold RA, Monaghan JJ. Smooth particle hydrodynamics: theory and applications to non-spherical stars. Mon Not R Astron Soc 1977; 181:375-89.

[14] Libersky LD, Petschek AG. Smooth particle hydrodynamics with strength of materials. Lecture Notes Phys 1990;395:248-57.

[15] Johnson GR, Stryk RA, Beissel SR. SPH for high velocity impact computations. Comput Methods Appl Mech Eng 1996;139:347-73.
[16] Shintate K, Sakine H. Numerical simulation of hypervelocity impacts of a projectile on laminated composite plate targets by means of improved SPH method. Composites: Part A 2004;35:683-92.

[17] Bonet J, Kulasegaram S. Correction and stabilization of smooth particle hydrodynamics methods with applications in metal forming simulations. Int J Numer Methods Eng 2000;47:1189-214.

[18] Gui YT, Liu GR. Meshless methods coupled with other numerical methods. Tsinghua Sci Technol 2005;10:8-15.

[19] Johnson GR. Linking of Lagrangian particle methods to standard finite element methods for high velocity impact simulations. Nucl Eng Des 1994;150:265-74.

[20] Attaway SW, Heinstein MW, Swegle JW. Coupling of smooth particle hydrodynamics with the finite element method. Nucl Eng Des 1994;150:199-205.

[21] Sauer M, Hiermeier S, Scheffer U. Modelling penetration events using FE/MLSPH adaptive coupling. In: 10th international symposium on interaction of the effect of munitions with structures, San Diego, California. 2001 May. p. 7-11.

[22] Hiermeier S, Peter J, Sauer M, Thoma K. Coupled FE-particle codes applied to material characterization and crash simulation. In: European Conference on Computational Mechanics (ECCM-2001), Cracow, Poland. 2001 June. p. 26-9.

[23] De Vuyst T, Vignjevic R, Campbell JC. Coupling between meshless and finite elements methods. Int J Impact Eng 2005;31:1054-64.

[24] Hiermaier S, Thoma K. Computational simulation of high velocity impact simulations using smoothed particle hydrodynamics. In: 9th DYMAT technical conference on materials and structural modelling in collosion research, TU, Munich, Germany, 1995.

[25] Hanssen AG, Langseth M, Hopperstad OP. Static and dynamic crushing of circular aluminium extrusions with aluminium foam filler. Int J Impact Eng 2000;24:475-507. 